\title{
Liquid crystal-filled meta-pixel with switchable asymmetric reflectance and transmittance
}

\author{
Oleksandr Buchnev ${ }^{\mathrm{a}, *}$, Nina Podoliak $^{\mathrm{b}}$ and Vassili A. Fedotov ${ }^{\mathrm{a}}$ \\ ${ }^{a}$ Optoelectronics Research Centre and Centre for Photonic Metamaterials, University of \\ Southampton, Southampton, SO17 1BJ, U.K. \\ ${ }^{b}$ Physics and Astronomy, University of Southampton, Southampton, SO17 1BJ, U.K. \\ *Corresponding author: O.Buchnev@ soton.ac.uk
}

\begin{abstract}
We fabricated and characterized a meta-pixel - metamaterial-based active optical device incorporating a twisted nematic liquid crystal cell. The meta-pixel exhibits directionally asymmetric transmittance and reflectance, which can be controlled electrically by realigning the liquid crystal. Apart from defining the optical response of the meta-pixel, the metamaterial also plays a vital role in implementing electrical control and alignment of the liquid crystal. Such a meta-pixel can find applications in a new generation of miniature displays, compact tunable optical filters and modulators.
\end{abstract}

\section{Introduction}

Metamaterials have attracted significant interest in photonics as a large class of manmade media with optical characteristics unavailable in nature [1,2]. They made possible such unique and exotic optical phenomena as negative refraction [3], asymmetric transmission [46], optical cloaking [7-9], slow light [10, 11], novel sources of coherent radiation [12, 13]; metamaterials enhanced polarization control [14-17] and absorption [18-20]. Systematic investigations of these fundamental phenomena have led to significant improvement of existing photonic devices and applications, in particular the broadband circular polarizer [21, 22], polarization spectral filter [23], and refractive-index sensor [24]. Integration of active metamaterials or parts of their structures into working optical components has led to a new concept of metadevices [25-28].

In this paper we demonstrate that a standard twisted nematic cell, when combined with a planar metamaterial, can be transformed into an optical metadevice. In this metadevice, 
which we named meta-pixel, the metamaterial structure replaces the liquid crystal alignment layer and one of the electrodes, while the twisted liquid crystal layer introduces electricallycontrolled asymmetry in the optical transmittance and reflectance of the metamaterial.

\section{Fabrication and Characterization}

The planar metamaterial was designed as a square array of $\mathrm{V}$-shaped plasmonic resonators connected along the rows of the array (see Fig. 1a). The resonators were formed by $80 \mathrm{~nm}$ wide gold nano-wires and placed with the period of $490 \mathrm{~nm}$, which rendered the resulting metamaterial nanograting as non-diffracting in the near infrared (and most of the visible) spectral range. The metamaterial covered the area of $25.6 \mu \mathrm{m} \times 24.2 \mu \mathrm{m}$ and was fabricated by focused ion beam milling (Helios Nanolab 600) in an $80 \mathrm{~nm}$ thick gold film deposited on a glass substrate. Each row of V-resonators was left electrically connected to the gold film on either end, as shown in Fig. 1a, enabling the application of an electrical potential to the entire metamaterial nanograting.

Metamaterial-based liquid crystal (MM-LC) meta-pixel was put together by combining the fabricated metamaterial nanograting with a top glass slide covered with a transparent ITO electrode and planar LC alignment layer (see Fig. 1b). The gap between the metamaterial and top glass slide was set to $12 \mu \mathrm{m}$ by silica bid spacers. The cell was filled with a highly birefringent nematic LC mixture 1825A with refractive indices of $n_{e}=1.90$ and $n_{o}=1.53$ [29]. As it was shown in the previous study [30], a gold zig-zag nano-grating aligns LC in the direction of the nano-wires stretch, i.e. along the rows of the nano-grating, with the strength of the alignment sufficient to even counteract stiction in nano-electro-mechanical systems [31]. The LC alignment at the top glass slide was therefore set in the direction perpendicular to the rows of the metamaterial nanograting. Such assembly created a $\pi / 2$ - twisted LC structure in the cell.

The transmittance and reflectance spectra of the MM-LC meta-pixel were measured in the near infrared spectral range (1.0 - $1.9 \mu \mathrm{m}$ wavelength) using a microspectrophotometer QDI2010 (CRAIC Technologies) with a sampling area of $10 \mu \mathrm{m} \times 10 \mu \mathrm{m}$. The polarization of light was switched between two orthogonal linear states, parallel and perpendicular to the rows of the metamaterial nanograting. The light was incident on the meta-pixel either from the metamaterial side or from the top glass side. 
(a)

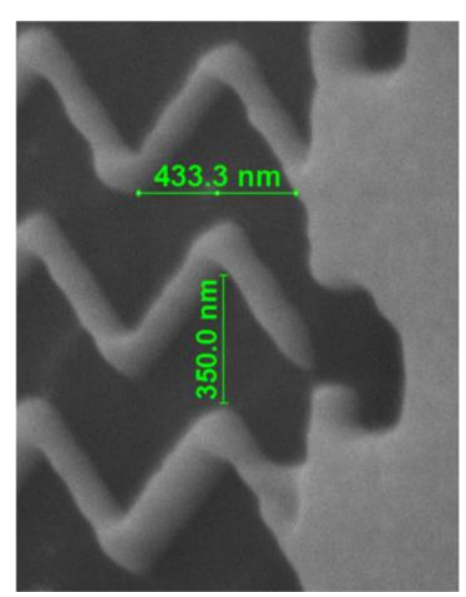

(b)

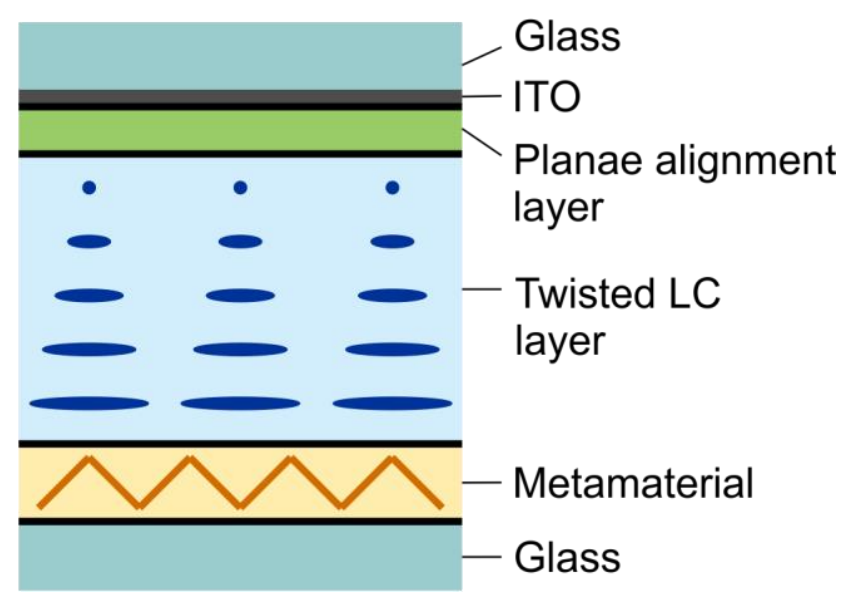

Fig. 1 (a) SEM image of fabricated V-shaped gold metamaterial array. The image shows an edge of the sample where the gold nano-wires are connected to the gold film. (b) Schematic of a MM-LC meta-pixel structure.

\section{Results and Discussion}

A planar metamaterial comprising $\mathrm{V}$-shaped plasmonic resonators demonstrates resonant, polarization-sensitive transmittance and reflectance in the near infrared part of the spectrum [30]. Incident light polarized perpendicular to the rows of the metamaterial nanograting (resonant polarization) can excite an electric dipole resonance, resulting from the $\lambda / 2$-mode of standing-wave plasma oscillations induced in the straight sections of V-resonators. The corresponding transmittance spectrum then demonstrates a clear stop-band centred at the resonance frequency, where incident light is reflected (as well as partly absorbed) by the metamaterial. Correspondingly, the reflection spectrum for the resonant polarization exhibits a peak at the resonance frequency. For the non-resonant polarization, the metamaterial is almost transparent within the near-infrared range of wavelengths with a nearly flat transmittance spectrum. No asymmetry in transmittance or reflectance depending on the direction of light propagation was observed in the metamaterial. The asymmetry in transmittance and reflectance is engineered by adding a twisted LC layer, which is able to rotate the polarization direction of ordinary and extraordinary waves by $\pi / 2$ if it is thick enough (i.e. satisfying the so-called Mauguin condition, $d \gg \frac{\lambda}{2 \Delta n}$, where $\lambda$ is the wavelength and $\Delta \mathrm{n}$ is the $\mathrm{LC}$ birefringence). 
The principle of asymmetric response in the MM-LC meta-pixel is schematically demonstrated in Fig 2. If a linearly polarized wave with resonant polarization and wavelength is incident on the meta-pixel from the metamaterial side (forward direction), it is reflected (and partly absorbed) by the metamaterial due to the excited resonance, exhibiting little transmission though the device (Fig. 2a). If, however, the same wave is incident from the other side of the meta-pixel (backward direction), the twisted liquid crystal layer rotates the polarization state of the wave by $\pi / 2$ before it reaches the metamaterial surface. The wave becomes non-resonantly polarized with respect to the metamaterial grating and, therefore, propagates through the device with little loss (Fig. 2a). The same asymmetric behaviour is observed for non-resonant polarization. In this case, the wave passes the meta-pixel if it propagates in the forward direction and is reflected (and partially absorbed) if it propagates in the backward direction (Fig. 2b).

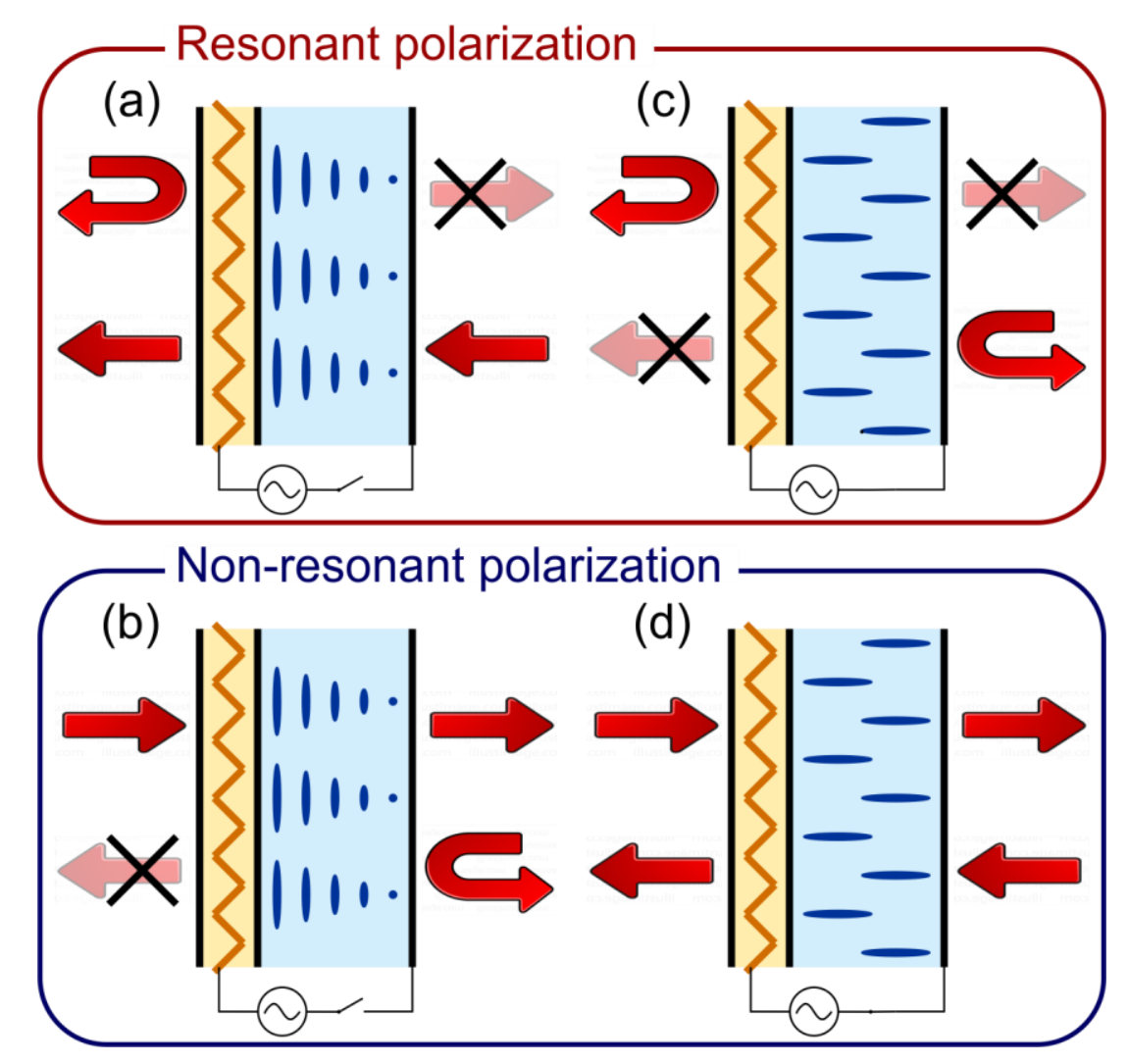

Fig. 2 Schematic of a MM-LC meta-pixel, demonstrating the principle of asymmetric transmittance and reflectance. The zig-zag orange line represents the metamaterial surface, the blue ellipses show the LC alignment direction, the red arrows depict the propagation direction of a wave at the resonant frequency. 
As the asymmetric transmittance/reflectance in the MM-LC meta-pixel connected with the twisted LC structure, this effect can be suppressed by realigning the LC with an electric field applied across the LC layer. For an applied voltage above the re-orientational threshold, the LC molecules align along the electric field forming a uniform homeotropic LC structure in the volume of the cell. This LC structure does not change the polarization state of a normally incident wave. In this case, the wave with the resonant polarization will not be transmitted by the meta-pixel regardless of the direction of propagation (Fig. 2c). Likewise, a wave with non-resonant polarization will be transmitted by the meta-pixel in both the forward and backward directions (Fig. 2d).

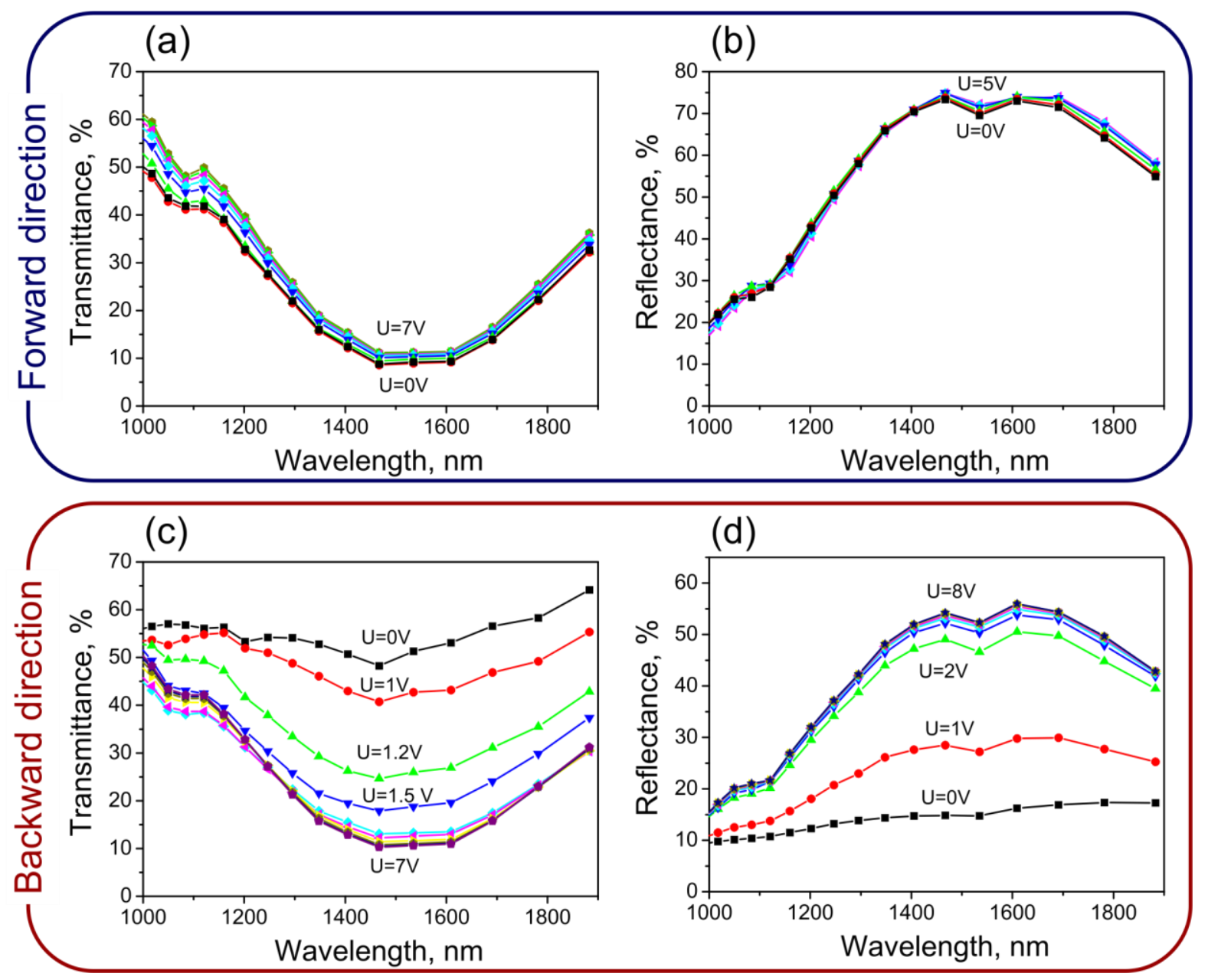

Fig. 3 (a)(c) Transmittance and (b)(d) reflectance spectra of the MM-LC cell for the forward and backward direction of propagation of a wave with resonant polarization at different voltages applied to the LC layer. 
Figure 3 shows asymmetric transmittance and reflectance, as well as switching between the asymmetric and symmetric regimes achieved in the MM-LC meta-pixel. The fabricated metamaterial array when combined with the LC layer exhibited a resonance around $1.5 \mu \mathrm{m}$, which can be seen as a minimum in the transmittance and a peak in the reflectance spectra measured for the forward propagation direction at zero voltage (Figs. 3 (a) and (b)). For the backward propagation direction at zero voltage, the transmittance and reflectance spectra are almost flat (Figs. 3 (c) and (d)), showing clearly the expected asymmetric behaviour. Some parasitic reflectance here (at the level of 10-15\%) is produced by the glass-air interface while the drop in overall transmittance below $85 \%$ is attributed to the material loss.

By applying a voltage across the LC layer, the switching from asymmetric to symmetric regime was observed. In the backward direction the transmittance/reflectance spectra were seen to change with increasing voltage. In particular, a stop-band in the transmittance spectra developed as the LC re-orientation switched from twisted to the homeotropic state. At the same time a peak in the reflectance spectra was observed (Figs. 3 (c) and (d)). In the forward direction the transmittance/reflectance changed very little upon applying a voltage, as evident from Figs. 3 (a) and (b). At the driving voltage exceeding $2 \mathrm{~V}$ the transmittance/reflectance spectra for the backward propagation direction almost matched the spectra for the forward direction, which signified the absence of the asymmetry. Lower reflectance peak value is related to propagation losses in the LC layer and double reflectance at the LC-glass interface. When the applied voltage was removed the LC layer relaxed back to the initial twisted state, and the asymmetric behaviour of the meta-pixel was restored.

\section{Conclusions}

We have demonstrated a meta-pixel - a photonic device consisting of a planar metamaterial and a twisted LC layer. The metamaterial defines the optical response of the meta-pixel, as well as serving as an LC alignment layer and an electrode. Combining the metamaterial with the twisted LC layer enables directional asymmetry in transmittance and reflectance of the device. The asymmetric behaviour can be suppressed (switched off) by reorienting the LCs from a twisted to a homeotropic structure with an externally applied electric field. The concept of the meta-pixel paves a way towards a new generation of compact switchers, modulators and miniature displays such as near-to-eye and virtual retina displays. 


\section{Acknowledgement}

The authors would like to thank Prof. Roman Dabrowski, Prof. Janusz Parka and Prof. Malgosia Kaczmarek for providing liquid crystal materials and stimulating discussions.

Our deepest acknowledgement is to the late Prof. Yuri Reznikov. Yuri's knowledge of, and his contribution to, the science of liquid crystals has rightly been acclaimed by the LC community across the world. One of us (Oleksandr Buchnev) had the good fortune to be Yuri's PhD student. He has often had cause to remember with gratitude Yuri's advice and guidance. This support was relevant not only in problems related to scientific research, but also for the far more mundane, but in some sense more personal, challenges thrown up by everyday life. Without any doubt, Yuri was a great scientist and a great man with a big heart, a heart which sadly stopped beating suddenly when he was still in the prime of life. Yuri's memory will be with us for many years to come.

\section{References}

1. E. Ozbay, "The magical world of photonic metamaterials," Opt. Photon. News 19(11), 22-26 (2008).

2. C. M. Soukoulis and M. Wegener, "Past achievements and future challenges in the development of three-dimensional photonic metamaterials," Nat. Photonics 5, 523$528(2011)$.

3. R. A. Shelby, D. R. Smith, and S. Schultz, "Experimental verification of a negative index of refraction," Science 292(5514), 77-79 (2001).

4. V. A. Fedotov, P. L. Mladyonov, S. L. Prosvirnin, A. V. Rogacheva, Y. Chen, and N. I. Zheludev, "Asymmetric propagation of electromagnetic waves through a planar chiral structure,” Phys. Rev. Lett. 97(16), 167401 (2006).

5. C. Menzel, C. Helgert, C. Rockstuhl, E.-B. Kley, A. Tünnermann, T. Pertsch, and F. Lederer, "Asymmetric transmission of linearly polarized light at optical metamaterials," Phys. Rev. Lett. 104(25), 253902 (2010).

6. A. V. Novitsky, V. M. Galynsky, and S. V. Zhukovsky, "Asymmetric transmission in planar chiral split-ring metamaterials: Microscopic Lorentz-theory approach," Phys. Rev. B 86(7), 075138 (2012). 
7. D. Schurig, J. J. Mock, B. J. Justice, S. A. Cummer, J. B. Pendry, A. F. Starr, and D. R. Smith, "Metamaterial electromagnetic cloak at microwave frequencies," Science 314(5801), 977-980 (2006).

8. T. Ergin, N. Stenger, P. Brenner, J. B. Pendry, and M. Wegener, "Three-dimensional invisibility cloak at optical wavelengths," Science 328(5976), 337-339 (2010).

9. H. Chen, C. T. Chan, and P. Sheng, "Transformation optics and metamaterials," Nat. Mater. 9(5), 387-396 (2010).

10. K. L. Tsakmakidis, A. D. Boardman, and O. Hess, “"Trapped rainbow' storage of light in metamaterials," Nature 450(7168), 397-401 (2007).

11. N. Papasimakis and N. I. Zheludev, "Metamaterial-induced transparency," Opt. Photon. News 20(10), 22-25 (2009).

12. N. I. Zheludev, S. L. Prosvirnin, N. Papasimakis, and V. A. Fedotov, "Lasing spaser," Nat. Photonics 2(6), 351-354 (2008).

13. O. Hess, J. B. Pendry, S. A. Maier, R. F. Oulton, J. M. Hamm, and K. L. Tsakmakidis, "Active nanoplasmonic metamaterials," Nat. Mater. 11(7), 573-584 (2012).

14. A. V. Rogacheva, V. A. Fedotov, A. S. Schwanecke, and N. I. Zheludev, "Giant gyrotropy due to electromagnetic-field coupling in a bilayered chiral structure," Phys. Rev. Lett. 97(17), 177401 (2006).

15. M. Decker, M. W. Klein, M. Wegener, and S. Linden, "Circular dichroism of planar chiral magnetic metamaterials," Opt. Lett. 32(7), 856-858 (2007).

16. J. Hao, Y. Yuan, L. Ran, T. Jiang, J. A. Kong, C. T. Chan, and L. Zhou, "Manipulating electromagnetic wave polarizations by anisotropic metamaterials," Phys. Rev. Lett. 99(6), 063908 (2007).

17. E. Plum, X. X. Liu, V. A. Fedotov, Y. Chen, D. P. Tsai, and N. I. Zheludev, "Metamaterials: optical activity without chirality," Phys. Rev. Lett. 102(11), 113902 (2009).

18. V. A. Fedotov, A. V. Rogacheva, N. I. Zheludev, P. L. Mladyonov, and S. L. Prosvirnin, "Mirror that does not change the phase of reflected waves," Appl. Phys. Lett. 88(9), 091119 (2006).

19. N. I. Landy, S. Sajuyigbe, J. J. Mock, D. R. Smith, and W. J. Padilla, "Perfect metamaterial absorber," Phys. Rev. Lett. 100(20), 207402 (2008).

20. K. Aydin, V. E. Ferry, R. M. Briggs, and H. A. Atwater, "Broadband polarizationindependent resonant light absorption using ultrathin plasmonic super absorbers," Nat Commun 2, 517-521 (2011). 
21. J. K. Gansel, M. Thiel, M. S. Rill, M. Decker, K. Bade, V. Saile, G. von Freymann, S. Linden, and M. Wegener, "Gold helix photonic metamaterial as broadband circular polarizer," Science 325(5947), 1513-1515 (2009).

22. Y. Zhao, M. A. Belkin, and A. Alù, "Twisted optical metamaterials for planarized ultrathin broadband circular polarizers," Nat Commun 3, 870-873 (2012).

23. N. I. Zheludev, E. Plum, and V. A. Fedotov, "Metamaterial polarization spectral filter: isolated transmission line at any prescribed wavelength," Appl. Phys. Lett. 99(17), 171915 (2011).

24. J. Zhao, Ch. Zhang, P. V. Braun, and H. Giessen, "Large-area low-cost plasmonic nanostructures in the NIR for Fano resonant sensing," Adv. Mater. (Deerfield Beach Fla.) 24(35), OP247-OP252 (2012).

25. N. I. Zheludev and Y. S. Kivshar, "From metamaterials to metadevices," Nat. Mater. 11(11), 917-924 (2012).

26. X. Fang, K. F. MacDonald, and N. I. Zheludev, "Controlling light with light using coherent metadevices: all-optical transistor, summator and invertor," Light Sci. Appl. 4, e292 (2015).

27. P. Cencillo-Abad, N. I. Zheludev, and E. Plum, "Metadevice for intensity modulation with sub-wavelength spatial resolution," Sci. Rep. 6, 37109 (2016).

28. P. Cencillo-Abad, E. Plum, E. T. F. Rogers, and N. I. Zheludev, "Spatial optical phase-modulating metadevice with subwavelength pixilation," Opt. Express 24(16), 18790-18798 (2016).

29. M. Reuter, N. Vieweg, B. M. Fischer, M. Mikulicz, M. Koch, K. Garbat, and R. Dabrowski, "Highly birefringent, low-loss liquid crystals for terahertz applications," APL Mater. 1, (2013), 012107 3-7.

30. O. Buchnev, J. Y. Ou, M. Kaczmarek, N. I. Zheludev, and V. A. Fedotov, "Electrooptical control in a plasmonic metamaterial hybridised with a liquid-crystal cell," Opt. Express 21 (2013), 1633-1638.

31. O. Buchnev, N. Podoliak, T. Frank, M. Kaczmarek, L. Jiang, and V. A. Fedotov, "Controlling stiction in nano-electro-mechanical systems using liquid crystals," ACS Nano 10 (2016), 11519-11524. 